\title{
Quantitative reversible one pot interconversion of three crystalline polymorphs by ball mill grinding
}

\author{
Ana M. Belenguer, ${ }^{[a]}$ Giulio I. Lampronti, ${ }^{*}{ }^{[a, b]}$ Adam A. L. Michalchuk, ${ }^{[c]}$ Franziska Emmerling, ${ }^{[c]}$ and \\ Jeremy K. M. Sanders ${ }^{*[a]}$
}

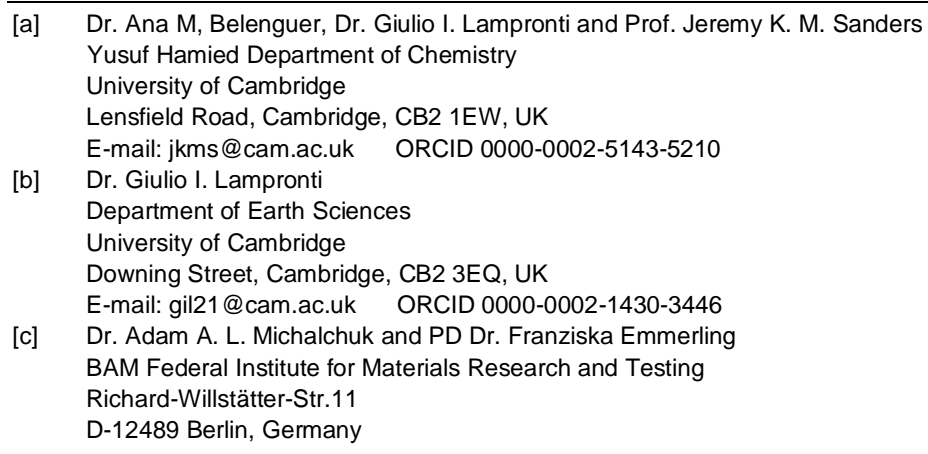

\begin{abstract}
We demonstrate here using a disulfide system the first example of reversible, selective and quantitative transformation between three crystalline polymorphs by ball mill grinding. This includes the discovery of a previously unknown polymorph. Each polymorph is reproducibly obtained under well-defined neat or liquid-assisted grinding conditions, revealing subtle control over the apparent thermodynamic stability. We discovered that presence of a contaminant as low as $1.5 \% \mathrm{~mol} \mathrm{~mol}^{-1}$ acting as a template is required to enable all these three polymorph transformations. The relative stabilities of the polymorphs are determined by the sizes of the nanocrystals produced under different conditions and by surface interactions with small amounts of added solvent. For the first time, we show evidence that each of the three polymorphs is obtained with a unique and reproducible crystalline size. This mechanochemical approach gives access to bulk quantities of metastable polymorphs that are inaccessible through recrystallisation.
\end{abstract}

The awareness in recent years that chemistry needs to become more sustainable, green, and cost effective has resulted in growing interest in mechanochemical methods. IUPAC in 2019 acknowledged mechanochemistry as one of the top ten emerging technologies in chemistry which will contribute to the well-being of society and the sustainability of planet Earth. ${ }^{[1]}$ We report here results that illustrate the power of ball grinding to discover and stabilize new, otherwise inaccessible polymorphs, and that shed light on the factors that lead to nanocrystalline polymorph stabilization.

Ball mill grinding is a common process for mechanochemical syntheses. In contrast to conventional solution-based reactions, it is atom-economic as the components are added in stoichiometric amounts, ${ }^{[2]}$ cost effective by generating products in shorter periods of time, ${ }^{[3]}$ and sustainable as it is performed solvent-free, and generally without heating. ${ }^{[4]}$ Moreover, mechanochemistry is considered 'green' as it does not produce solvent or toxic chemical waste..$^{[5]}$ In addition to environmental benefits, ball mill grinding circumvents issues of solubility. ${ }^{[6]}$ In many cases, mechanochemical processing allows access to unexpected reaction pathways, some of which even yield new products, inaccessible by solution routes. ${ }^{[7]}$ These many advantages have made mechanochemistry an attractive avenue for research, with applications in many organic,,$^{[8]}$ inorganic ${ }^{[9]}$ and metal-organic chemical syntheses ${ }^{[10]}$, to prepare functional materials ${ }^{[11]}$ and to generate nanocatalysts designed for biomass conversion and bio-based catalysts ${ }^{[12]}$.

Ball mill grinding reactions can be performed under neat conditions (NG) and under liquid assisted grinding (LAG) conditions, wherein a sub-stoichiometric amount of liquid is added to the powder. This addition of liquid often leads to faster reactions and to a different outcome as in the formation of a different polymorph of the product. ${ }^{[13]}$ Polymorphism is a property inherent to the solid state. It is the ability of a compound to exist in multiple solid forms differing in relative intermolecular and/or interatomic distances. Such crystal properties determine physicochemical properties such as melting point, dissolution behaviour, solubility, reactivity, bioavailability and stability against physical and chemical stress, making the topic of understanding how different polymorphs can be reliably prepared of major interest especially to the pharmaceutical industry. ${ }^{[14]}$ Many cocrystals and salts that are inaccessible by solution methods have been prepared by ball mill grinding; in some cases various polymorphs have been reported. In most cases only two polymorphs have been prepared by ball mill grinding. ${ }^{[15]}$

Only a handful of examples of the full or partial preparation of three polymorphic forms of the same substance (crystalline or amorphous) by ball mill grinding have been published to date: anthranilic acid, ${ }^{[16]} \mathrm{Y}$-aminobutyric acid (GABA), ${ }^{[17]} \mathrm{N}$-acetyl-I-phenylalanyl-amino, ${ }^{[18]}$ Praziquantel, ${ }^{[19]} 1: 1$ caffeine-anthranilic acid cocrystal, ${ }^{[20]}$ and 1:1 salt of vinpocetine oxalate. ${ }^{[21]}$ Polymorph conversion by ball mill grinding has been an area of interest, as it enables the formation of bulk quantities of metastable polymorphs by alternative techniques to traditional ones, ${ }^{[16]}$ though often not accessible by classical solution methods. ${ }^{[17-19]}$ Recently, Arhankelskis et al. demonstrated reversibility between two crystalline and one amorphous form of the 1:1 salt of vinpocetine oxalate. ${ }^{[22]}$ Only partial reversibility of polymorph transformation between pairs of polymorphs has been shown in those cases where three crystalline polymorphs were obtained by ball mill grinding. ${ }^{[16,18,20]}$ Full reversibility between two polymorphs by ball mill grinding has been shown for a number of molecular compounds, via "one pot polymorph turnover experiments". ${ }^{[2,2]}$ In these experiments, the same sample powder is converted from one polymorph to the other and back by changing the milling conditions. This means that the milling jar is opened to allow for the 
LAG solvent to be removed by evaporation and for analyses to characterize the milled powder, but the powder itself is not replaced. We believe that full reversibility of polymorph conversions by mechanochemical methods will prove to be a common and general phenomenon. For this reason, there should be no limit in principle to the number of polymorphs that can be reversibly transformed by such turnover experiments.

We present here for the first time the one pot reversible and quantitative interconversion of three crystalline polymorphs of a compound by ball mill grinding at milling equilibrium, Scheme 1 . The fact that all three polymorphs are crystalline proves unambiguously that the product of ball milling is uniquely and reproducibly defined by the milling conditions. Our model disulfide compound, 2nitrophenyl-4-chlorophenyl-disulfide, here called 1-2, has been investigated by our team for various purposes together with a few related phenyl-disulfide compounds with different functional groups on the phenyl ring. ${ }^{[2,13,22-23]}$ The number "1" refers to the 2-nitrophenylpart, and (2-nitrophenyl) $)_{2}$-disulfide is thus called 1-1; while the number "2" corresponds to the 4-chlorophenyl- part, and (4chlorophenyl) 2 -disulfide is thus called 2-2. Form $\mathbf{B}$ is the bulk thermodynamic polymorph of $\mathbf{1 - 2}$ under ambient temperature and pressure, and can be easily recrystallized from a range of solvents (see ESI Section 3.5 for details). Recrystallized crystals of Form B were used for the polymorph turnover experiments presented in this paper. Form $\mathbf{A}$ is obtained by milling under NG conditions from Form B or the newly-discovered Form C while Form B can be obtained from Form A under LAG with a range of solvents, Scheme ${ }_{1 .}{ }^{[22]}$ and from Form $\mathbf{C}$ by LAG with MeCN. Form $\mathbf{C}$ can be obtained from Form $\mathbf{B}$ or Form $\mathbf{A}$ by LAG with water. Details of the crystal structure solution of Form C, introduced for the first time in this paper, are reported in the ESI Section 2.2.

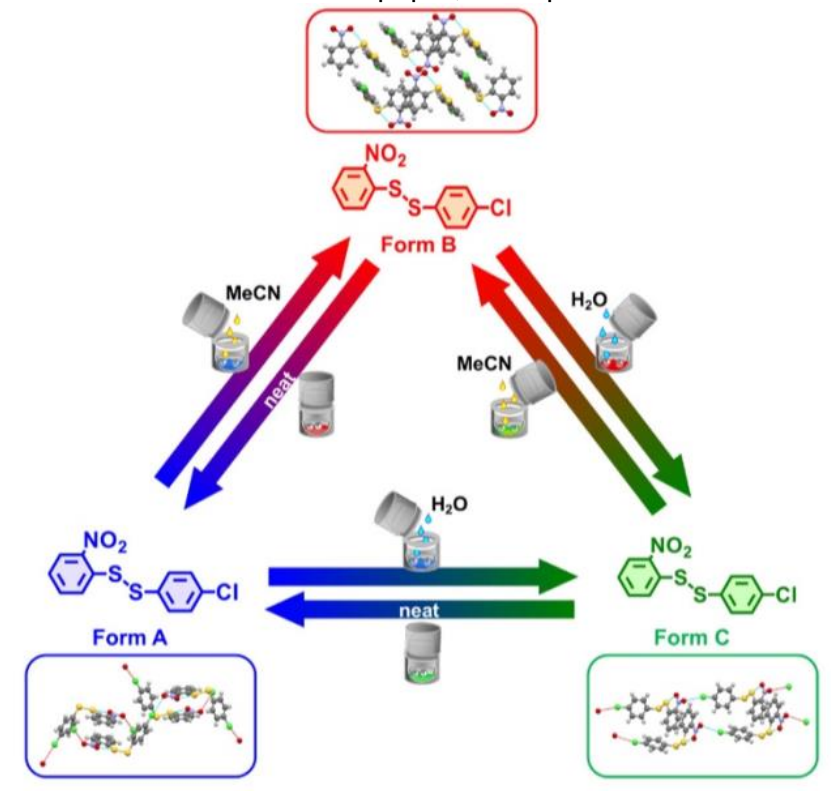

Scheme 1. Polymorph interconversion between the three polymorphs of the molecule 1-1 by ball mill grinding until equilibrium is achieved. LAG stands for liquid assisted grinding (addition of a few drops of solvents). NG stands for neat grinding in the absence of added solvent. The outer clockwise arrows indicate the in-situ polymorph interconversion from Form B $\rightarrow$ Form $\mathbf{C} \rightarrow$ Form A $\rightarrow$ Form B while the inner anticlockwise arrows indicate the one pot polymorph transformation in the reverse direction. The crystal structures for each polymorph are illustrated.

We can quantitatively interconvert these three polymorphs (Form A, Form B and previously unknown Form C) by modifying the experimental ball mill grinding conditions using NG or LAG. Figure 1 a) shows this polymorph transformation in an arbitrarily chosen clockwise direction. Starting from crystals of Form B, LAG with water results in Form C; NG of Form C results in Form A; LAG of Form A with acetonitrile returns to Form B. Figure $1 \mathrm{~b}$ ) show this polymorph transformation in an anticlockwise direction, transforming the crystals of Form B by NG to Form A; this polymorph is subjected to LAG with water to obtain Form $\mathbf{C}$ which on LAG with acetonitrile returns to Form B. We ran both clockwise and anticlockwise turnover experiments through two cycles and in duplicate (see SI Section 5 and Figure 2) to prove that these polymorph transformations can be repeated as many times as desired and are reproducible.

There is one caveat. All these polymorphic transformations are only possible when a small amount of 1-1 is either present or is added to the powder as an impurity. The amount of 1-1 present or added to the powder of the polymorph turnover cycles displayed in Figure 1 is $1.5 \% \mathrm{~mol} \mathrm{~mol}^{-1}$ (see ESI Section 4 for further experimental details and comments). This shows the importance of exceptionally small amounts of contaminants in enabling polymorph transformations by ball mill grinding. This level of contaminant is present in many chemicals, including those that are commercially available, and this phenomenon may be much more widespread than generally realised. Such small concentration of impurities is virtually undetectable by PXRD and was thus identified and quantified by high-performance liquid chromatography (HPLC, see ESI Section 2) only, which shows that a multitechnique approach is crucial in these solid state investigations. The role of this 1-1 contaminant in the polymorph conversion is puzzling. One possible explanation is that such impurity alters the relative polymorph stability by substituting the heterodimer in its crystal structures, akin to forming a solid solution, ${ }^{[24]}$ but such hypothesis is largely excluded by accurate PXRD analyses which do not indicate molecular substitution within any of the three polymorphs (see ESI Section 2.2 for details). We believe instead that the contaminant acts as a template for the nucleation of the different polymorphs. Importantly, as no base catalyst was used in these experiments, ${ }^{[13,22-23,23 c-9]}$ no covalent bonds are expected to be broken during the turnover cycles displayed in Figure 1. The chemical composition is therefore conserved throughout the entire 
three-polymorph turnover cycle and cannot explain the change in relative polymorph stability under the different milling conditions used or the reversibility of the process.

In their bulk forms, dispersion corrected density functional theory simulations suggest the polymorphic stability to follow as Form B > Form C > Form A (ESI Section 7). Notably, the relative stability of Forms B and C derive from entropic contributions, which can be easily affected by small changes in temperature. We however note that our simulations suggest that no re-ordering of the polymorphic phases occurs with temperature. Instead, the ability to reproducibly and selectively obtain each polymorphic form can be rationalized by considering crystallite sizes generated by milling under different conditions. We have shown previously that for nanocrystalline materials, polymorph stabilities depend not only on internal lattice energy, but also on crystal size and surface solvation effects. ${ }^{[22]}$ Ball mill grinding breaks crystals down to nanometre length scales, with a dramatic increase in their surface to volume ratio. We previously performed polymorph interconversion turnover experiments between Form A and Form B, showing that the crystal size (Scherrer size) of Form A was consistently smaller (around $40 \mathrm{~nm}$ ) as compared to Form B $(60-79 \mathrm{~nm})$. $^{[22]}$ Once crystals reach this nano scale, a significant proportion of their molecules are located on the surface of the crystal, with a lower number of stabilizing intermolecular interactions as compared with molecules in the bulk. We believe that the presence of additives (e.g. liquid) acts to stabilize these surfaces, ${ }^{[22,25]}$ thereby altering the relative stability of nanocrystals, and therefore with the potential to cause a polymorph conversion. ${ }^{[22]}$ These fundamental concepts are universal and apply to all chemistries.
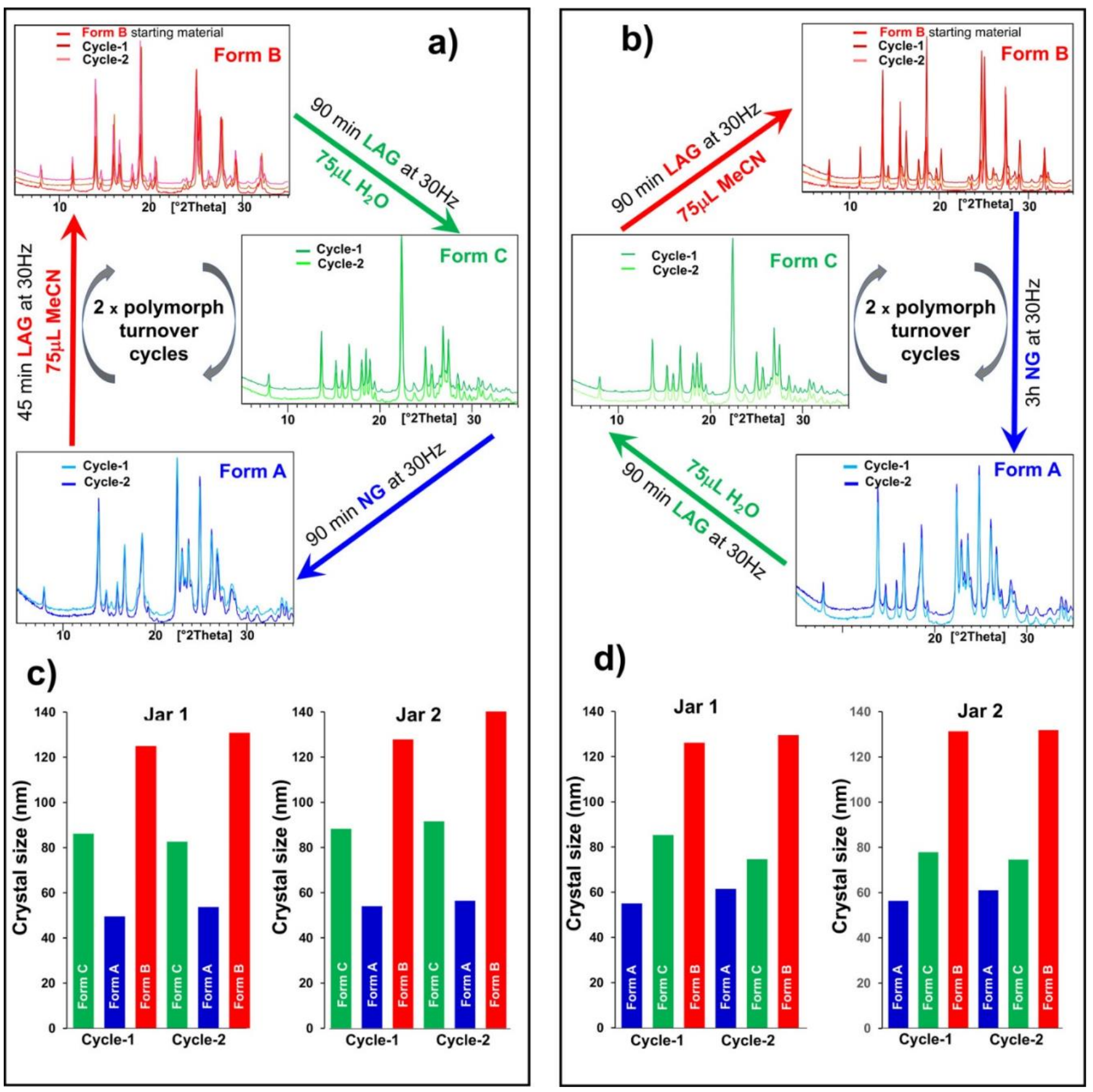
Figure 1. One pot polymorph transformation turnover experiments starting from $300 \mathrm{mg}$ of Form $\mathbf{B}$ crystals containing $1.5 \% \mathrm{M}$ of $\mathbf{1 - 1}$ from the recrystallisation procedure or purposely added. a) clockwise direction from Form $\mathbf{B} \rightarrow$ Form $\mathbf{C} \rightarrow$ Form $\mathbf{A} \rightarrow$ Form $\mathbf{B}$; b) counter-clockwise direction from Form $\mathbf{B} \rightarrow \mathbf{F o r m} \mathbf{A} \rightarrow$ Form $\mathbf{C} \rightarrow$ Form B. The different experimental conditions are documented on the diagrams. The duplicate experiments (jar 2) to a) and b) are in ESI Section 5 . $\mathbf{C}$-d) Crystal size for Form B, Form A and Form C obtained in the one-pot polymorph turnover cycles a) and b) respecively. Each clock-and anticlockwise polymorph turnover cycle was performed in duplicate (jar 1 and jar 2).

Estimates via the Scherrer equation revealed that at least one of the two polymorphs involved in the previous two-polymorph turnover experiments that we studied had a crystal size smaller than $100 \mathrm{~nm} \cdot{ }^{\left[{ }^{[2]}\right.}$ Analogously, Form A and Form C have crystal sizes in the order of tens of nanometers. Moreover, the crystal sizes of all three polymorphs (Form A, Form B and Form C) are also highly reproducible, independently of whether they are obtained in the clockwise or anticlockwise turnover cycle. Consistently, Form A has the smallest crystals $(50 \mathrm{~nm})$ while Form B crystals are the largest $(140 \mathrm{~nm})$ (Figure $1 \mathrm{c})$ and d)). This experimental observation hints at a difference of the bulk:surface energy balance across the three polymorphs. Moreover, the liquid used under LAG conditions must interact with crystal surfaces affecting surface stabilities as a consequence. The polymorph stability order is thus influenced by the solvent nature and the concentration used as well as the crystal size and morphology.

Conceptually, each polymorph can be thought of as representing a local thermodynamic well, accessible under the specific set of milling conditions. This is demonstrated by the fact that these polymorph conversions can be reversed by changing the milling conditions. Moreover, our computational studies (ESI Section 7) show an inverse correlation between the polymorph lattice energies and the experimental crystal size obtained by the ball mill grinding. This strongly suggest that different polymorphic forms must grow to different sizes before 'bulk' properties dominate their stability, consistent with earlier findings. ${ }^{[22]} \mathrm{A}$ thorough understanding of nano-scale stability will clearly be critical for determining the outcome of ball milling transformations.

a)
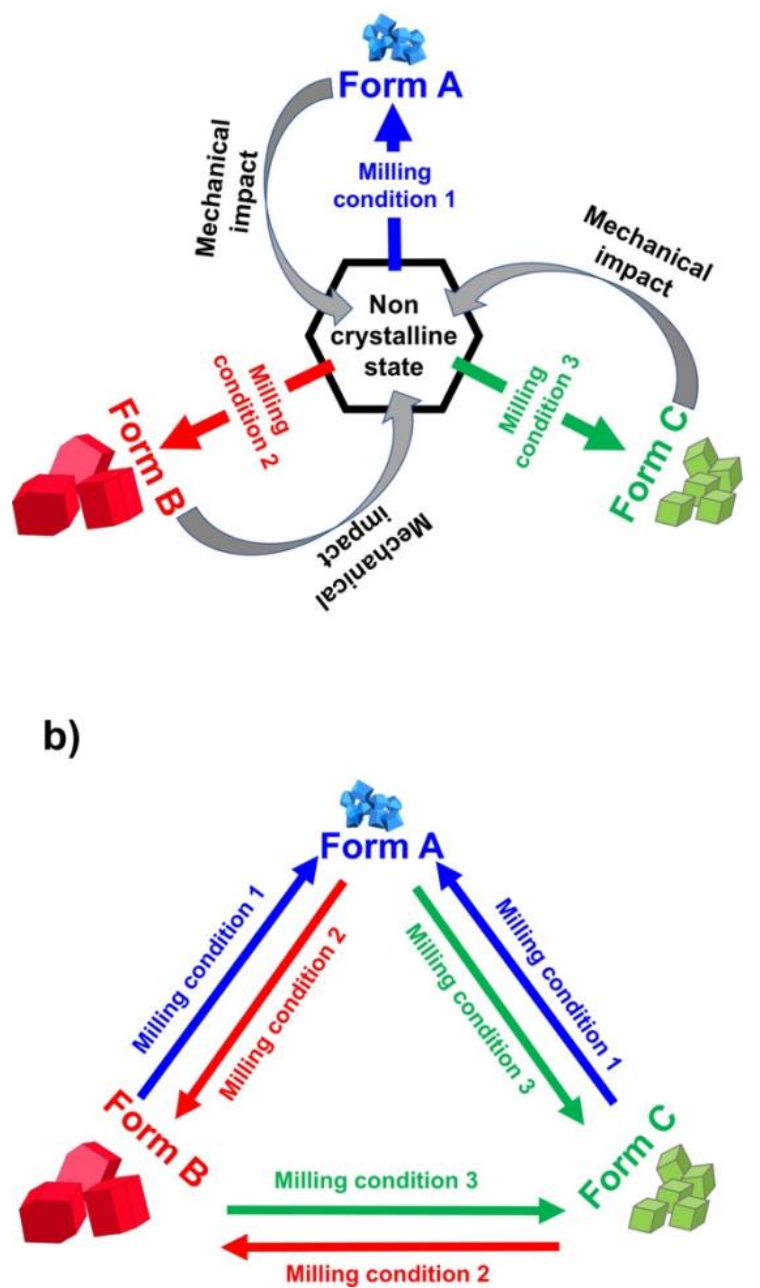

Figure 2. Two hypotheses for the polymorph conversion pathways: a) bottom-up nucleation and growth via a non-crystalline state; b) top-down single crystal to single crystal transformation. See text for discussion.

It is clear the nanoscale crystal properties are responsible for determining the result of polymorphic transformations under ball milling conditions. However, it is not yet clear whether these polymorph interconversions: (a) occur via some reconstructive mechanism, dominated by nucleation and growth; or (b) are of a single crystal to single crystal transformation (see Figure 2). In the latter case, the 
crystallites of one polymorph approach a threshold crystal size under the specific milling conditions beyond which a phase transformation of displacive nature occurs towards a more stable polymorph. In the former case, the polymorph is dictated by which nuclei are stabilised (or destabilised) under the given milling conditions. It is subsequently this nucleus that grows to the size that we

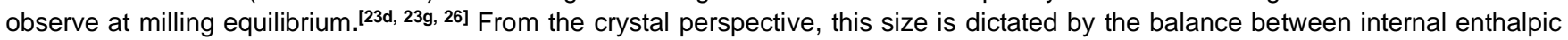
stabilisation and the destabilising effects of a growing surface. However, under ball milling conditions crystal growth and crystal breaking by the ball milling presumably play a dominant role in the final crystal size. Our previous work has suggested that, within a small range (ca. $15-30 \mathrm{~Hz}$ ), milling frequency does not alter the crystal size at milling equilibrium. ${ }^{[23 \mathrm{~d}]}$ Although we might expect in principle the equilibrium to be sensitive to both milling frequency and energy, we suggest that the parameter range accessible within a conventional ball mill is too narrow to make this effect observable. ${ }^{[27]}$ This suggests that milling equilibria obtained in this way are insensitive to small changes in milling conditions, making them robust and transferable. This is will be important for the translation of academic research into industrial settings.

In conclusion, we have reported the first one pot quantitative and reversible polymorph turnover cycle between three polymorphs of a molecular compound by ball mill grinding: Form A can be obtained from either Form B or Form C under NG conditions; Form B can be obtained from either Form A or Form C under LAG conditions with MeCN; Form C can be obtained from either Form A or Form B under LAG conditions with water. We have shown that a contaminant is critical to enable polymorph transformations under ball milling conditions, even at the low concentration levels typical of commercially available chemicals. We have given evidence of the exceptional reproducibility and specificity of the size for each of the three polymorphs. XRD analyses of specimens of different polymorph transformation cycles, show that Form A has reproducibly the smallest crystal size $(50 \mathrm{~nm})$ while Form B is the largest ( $140 \mathrm{~nm})$ of the three polymorphs. These experimental results are further proof that polymorph stabilities under ball mill grinding conditions are affected by crystal size and surface effects such as the stabilization or destabilization caused by interactions between the solvent molecules and the nanocrystal surfaces. Furthermore, we have described a newly-discovered polymorph (Form $\mathbf{C}$ ) which to date has been prepared only via ball mill grinding; this may prove a productive general route for the discovery of new polymorphs of significant molecular entities.

\section{Orcid}

Dr. Ana M, Belenguer

Dr. Giulio I. Lampronti

0000-0002-0443-4856

0000-0002-1430-3446

Dr. Adam A. L. Michalchuk 0000-0001-7405-3269

PD Dr. Franziska Emmerling 0000-0001-8528-0301

Prof. Jeremy K. M. Sanders $\quad$ 0000-0002-5143-5210

\section{Institute and/or researcher Twitter usernames}

\section{Ana Belenguer@@Amb84Ana \\ Adam Michalchuk@@amichalchuk \\ Franziska Emmerling @franemmerling}

Yusuf Hamied Department of Chemistry, University of Cambridge

Department of Earth Sciences, University of Cambridge

BAM Federal Institute for Materials Research and Testing @BAMresearch

COST action 18112: 'Mechanochemistry for Sustainable Industry'

\author{
@ChemCambridge \\ @EarthSciCam
}

@MechSustlnd

\section{Acknowledgements}

Experiments were conducted at the Yusuf Hamied Department of Chemistry and Department of Earth Sciences, University of Cambridge, and at BAM (Federal Institute for Materials Research and Testing) in Berlin. AMB, AALM and FE thank D. Al-Sabbagh for support with PXRD at BAM. In Cambridge Chemistry we thank C. Truscott for support of PXRD, C. A. Bland and P. Donnelly for the mechanical and software design of the automation of the grinders for repeat grinding, the mechanical workshop team for the manufacture of the jars and the Chris Hunter research, for general support. G.I.L. thanks the Department of Earth Sciences for general support. The authors acknowledge COST Action CA18112 - Mechanochemistry for Sustainable Industry.

Keywords: mechanochemistry $\bullet$ ball mill grinding $\bullet$ polymorphism $\bullet$ reversible polymorph interconversion $\bullet$ nanocrystals 


\section{Table of Contents}

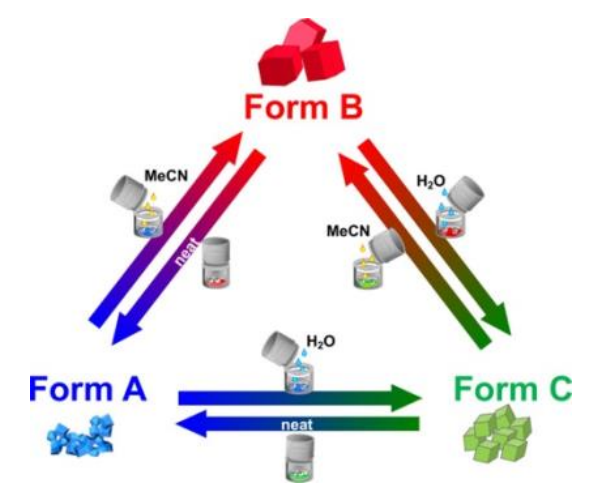

The first time one pot reversible and quantitative interconversion of three crystalline polymorphs of a compound by ball mill grinding to milling equilibrium is here presented. A contaminant in a concentration as low as $1.5 \% \mathrm{~mol} \mathrm{~mol}^{-1}$ is required to enable all these three polymorph transformations. We propose the contaminant acts as a template for the polymorph conversion. The relative stabilities of the polymorphs must depend on the size of the nanocrystallites produced under different conditions and by surface interactions with small amounts of added solvent.

[1] F. Gomollón-Bel, in Chemistry International, Vol. 41, 2019, p. 12.

[2] A. M. Belenguer, A. J. Cruz-Cabeza, G. I. Lampronti, J. K. M. Sanders, CrystEngComm 2019, 21, $2203-2211$.

[3] T.-X. Métro, J. Martinez, F. Lamaty, ACS Sustainable Chemistry \& Engineering 2017, 5, 9599-9602.

[4] aO. Galant, G. Cerfeda, A. S. McCalmont, S. L. James, A. Porcheddu, F. Delogu, D. E. Crawford, E. Colacino, S. Spatari, ACS Sustainable Chemistry \& Engineering 2022; bS. L. James, C. J. Adams, C. Bolm, D. Braga, P. Collier, T. Friscic, F. Grepioni, K. D. M. Harris, G. Hyett, W. Jones, A. Krebs, J. Mack, L. Maini, A. G. Orpen, I. P. Parkin, W. C. Shearouse, J. W. Steed, D. C. Waddell, Chemical Society Reviews 2012, 41, 413-447.

[5] L. Konnert, A. Gauliard, F. Lamaty, J. Martinez, E. Colacino, ACS Sustainable Chemistry \& Engineering 2013, 1, $1186-1191$.

[6] T. Seo, N. Toyoshima, K. Kubota, H. Ito, Journal of the American Chemical Society 2021, 143, 6165-6175.

[7] R. A. Haley, A. R. Zellner, J. A. Krause, H. Guan, J. Mack, ACS Sustainable Chemistry \& Engineering 2016, 4, $2464-2469$.

[8] D. Margetic, V. Strukil, Mechanochemical Organic Synthesis, Elsevier, 2016, ISBN: 978-0-12-802184-2.

[9] D. Tan, F. García, Chemical Society Reviews 2019, 48, 2274-2292.

[10] C.-A. Tao, J.-F. Wang, Crystals 2021, 11, 15.

[11] B. Szczęśniak, S. Borysiuk, J. Choma, M. Jaroniec, Materials Horizons 2020, 7, 1457-1473.

[12] C. M. Cova, R. Luque, BMC Chemical Engineering 2019, 1, 16.

[13] A. M. Belenguer, T. Friscic, G. M. Day, J. K. M. Sanders, Chem. Sci. 2011, 2, 696-700

[14] aA. J. Cruz-Cabeza, N. Feeder, R. J. Davey, Communications Chemistry 2020, 3, 142; bSarah L. Price, Faraday Discussions 2018, 211, 9-30.

[15] aM. Solares-Briones, G. Coyote-Dotor, J. C. Páez-Franco, M. R. Zermeño-Ortega, C. M. de la O Contreras, D. Canseco-González, A. Avila-Sorrosa, D. Morales-Morales, J. M. Germán-Acacio, Pharmaceutics 2021, 13, 790; bD. Hasa, W. Jones, Advanced Drug Delivery Reviews 2017, 117, 147-161.

[16] A. V. Trask, N. Shan, W. D. S. Motherwell, W. Jones, S. Feng, R. B. H. Tan, K. J. Carpenter, Chemical Communications 2005, 0, 880-882.

[17] L. Wang, G. Sun, K. Zhang, M. Yao, Y. Jin, P. Zhang, S. Wu, J. Gong, ACS Sustainable Chemistry \& Engineering 2020, 8, 1678116790 .

[18] B. D. Altheimer, S. Pagola, M. Zeller, M. A. Mehta, Cryst. Growth Des. 2013, 13, 3447-3453.

[19] B. Saikia, A. Seidel-Morgenstern, H. Lorenz, Cryst. Growth Des. 2021, 21, 5854-5861.

[20] D. Hasa, E. Miniussi, W. Jones, Cryst. Growth Des. 2016, 16, 4582-4588.

[21] M. Arhangelskis, D.-K. Bučar, S. Bordignon, M. R. Chierotti, S. A. Stratford, D. Voinovich, W. Jones, D. Hasa, Chem. Sci. 2021, 12 3264-3269.

[22] A. M. Belenguer, G. I. Lampronti, A. J. Cruz-Cabeza, C. A. Hunter, J. K. M. Sanders, Chem. Sci. 2016, 7, 6617-6627.

[23] aA. M. Belenguer, G. I. Lampronti, D. J. Wales, J. K. M. Sanders, Journal of the American Chemical Society 2014, 136, 1615616166; bA. M. Belenguer, G. I. Lampronti, N. De Mitri, M. Driver, C. A. Hunter, J. K. M. Sanders, Journal of the American Chemical Society 2018, 140, 17051-17059; cA. M. Belenguer, G. I. Lampronti, J. K. M. Sanders, JoVE 2018, e56824; dAna M. Belenguer, A. A. L. Michalchuk, G. I. Lampronti, J. K. M. Sanders, Beilstein J. Org. Chem. 2019, 15, 1226-1235; eA. M. Belenguer, G. I. Lampronti, J. K. M. Sanders, Israel Journal of Chemistry, n/a; fA. M. Belenguer, A. A. L. Michalchuk, G. I. Lampronti, J. K. M. Sanders, ChemSusChem, n/a, e202102416; gG. I. Lampronti, A. A. L. Michalchuk, P. P. Mazzeo, A. M. Belenguer, J. K. M. Sanders, A. Bacchi, F. Emmerling, Nature Communications 2021, 12, 6134.

[24] W. Kras, A. Carletta, R. Montis, R. A. Sullivan, A. J. Cruz-Cabeza, Communications Chemistry 2021, 4, 38.

[25] aH. Zhang, B. Gilbert, F. Huang, J. F. Banfield, Nature 2003, 424, 1025-1029; bA. Navrotsky, L. Mazeina, J. Majzlan, Science 2008, 319, 1635-1638.

[26] T. Friščić, I. Halasz, P. J. Beldon, A. M. Belenguer, F. Adams, S. A. J. Kimber, V. Honkimäki, R. E. Dinnebier, Nat. Chem. 2013, 5, 66-73. 

aK. Hara, E. Yamasue, H. Okumura, K. Ishihara, Journal of Alloys and Compounds - J ALLOYS COMPOUNDS 2011, 509; bY. Iguchi, M. Senna, Powder Technology 1985, 43, 155-162. 